\title{
Multi-objective System Design Optimization via PPA and a Fuzzy Method
}

\author{
Mohamed Arezki Mellal ${ }^{1,2}$ (i) $\cdot$ Abdellah Salhi $^{3}$
}

Received: 19 December 2019/Revised: 1 January 2021/Accepted: 9 February 2021/Published online: 9 April 2021

(C) The Author(s) 2021

\begin{abstract}
System design deals with various challenges of targets and resources, such as reliability, availability, maintainability, cost, weight, volume, and configuration. This paper deals with the multi-objective system availability and cost optimization of parallel-series systems by resorting to the multi-objective strawberry algorithm also known as the Plant Propagation Algorithm or PPA and a fuzzy method. It is the first implementation of this optimization algorithm in the literature for this kind of problem to generate the Pareto Front. The fuzzy method allows helping the decision maker to select the best compromise solution. A numerical case study involving 10 subsystems highlights the applicability of the proposed approach.
\end{abstract}

Keywords Multi-objective system design - Availability · Cost $\cdot$ Parallel-series system · Plant Propagation

Algorithm $\cdot$ Fuzzy method

\section{Introduction}

Resource allocation is one of the most commonly used methods in system design and exploitation. It allows optimally using available resources and respecting product

Mohamed Arezki Mellal

mellal.mohamed@gmail.com; mellal.mohamed@univ-

boumerdes.dz; mamellal@umd.edu

1 LMSS, Faculty of Technology, M'Hamed Bougara University, 35000 Boumerdes, Algeria

2 Center for Advanced Life Cycle Engineering (CALCE), University of Maryland, College Park, MD 20742, USA

3 Department of Mathematical Sciences, University of Essex, Colchester, UK specifications. Industrial plants consist of many components and their system dependability (RAMS + C: reliability, availability, maintainability, safety, and cost) should be optimized [1-5]. In the last few decades, efforts have been devoted to the question of optimal allocation in system dependability. These efforts may be divided into two categories depending on the nature of the formulated problems: single objective and multi-objective optimization problems. Most solution approaches are based on bioinspired optimization techniques, also called soft computing methods or artificial intelligence methods, as these methods proved their effectiveness in practice.

In [6], the reliability of parallel-series systems has been optimized by the redundancy allocation with component choices under the constraints of cost and weight. Several authors proposed solution approaches for the optimal system reliability-redundancy allocation subject to cost, volume, and weight [7-13]. Chen [7] and Hsieh and You [8] developed immune algorithms. Garg et al. [9] and Garg [10] used the artificial bee colony and cuckoo search, respectively. Mellal and Zio implemented a penalty guided stochastic fractal search for 10 case studies in [11], whereas a pharmaceutical plant consisting of 10 subsystems connected in series has been investigated in [12] using the above algorithm, the cuckoo optimization algorithm with penalty function, and the genetic algorithm. A large-scale reliability-redundancy allocation problem has been solved in [13] using the cuckoo optimization algorithm, particle swarm optimization, and the genetic algorithm. The optimization problem of the reliability-redundancy allocation has been simplified in [14] by resorting to the theory of survival signature. On the other hand, Juybari et al. [15] and Mellal and Zio [16] considered the cold-standby strategy for the redundant components. Chebouba et al. [17] considered the reliability and cost as objectives under 
data uncertainties. In [18-20], the goal was to allocate the optimal number of redundant components, failure rates and repair rates in each subsystem for maximizing the system availability under the cost limit and design constraints. The author of $[18,19]$ used the Tabu-genetic algorithm, whereas in [20] the authors used five optimization techniques: cuckoo optimization algorithm, genetic algorithms, flower pollination algorithm, differential evolution, and particle swarm optimization. The study showed that the cuckoo optimization algorithm provided better results. In $[21,22]$, the authors considered both the system availability and the cost function described in [18-20] as objective functions using the weighted sum methods and the non-dominated sorting genetic algorithm II (NSGA-II), respectively. The bi-criteria problem has been tackled by converting it into a single objective optimization one using two weighted sum methods. The system cost under availability constraint with failure dependencies has been optimized in $[4,23]$ using an adaptive cuckoo optimization algorithm and the genetic algorithm, respectively. The optimum number of redundant components and repair teams have been allocated in each subsystem for this purpose.

The aim of the present work is to consider the conflicting and nonlinear objectives of availability and cost of parallel-series systems when redundancy, failure rate, and repair rate allocations are considered as design variables. An implementation of a multi-objective optimization algorithm, called multi-objective strawberry algorithm, is presented in order to generate the Pareto front. A fuzzy method is applied to select the best compromise solution for the decision maker. The remainder of the paper is organized as follows: Sect. 2 describes the multi-objective optimization problem. Section 3 presents the principles of the multi-objective strawberry algorithm. Section 4 highlights the method applied for selecting the best compromise solution. A numerical case study with results and discussion are illustrated in Sects. 5 and 6, respectively. Finally, the last section concludes the paper.

\section{Problem Description}

The design of the parallel-series system shown in Fig. 1 when considering the redundancy, failure rate and repair rate allocations as design variables is given as follows [18-21]:

\section{System cost}

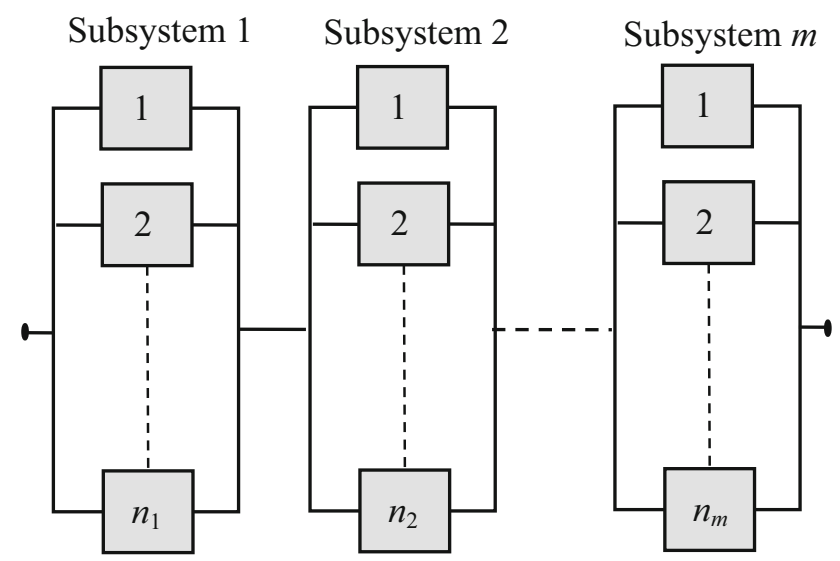

Fig. 1 Parallel-series system

$$
C_{\mathrm{s}}(n, \lambda, \mu)=\sum_{i=1}^{m}\left[\left(\alpha_{i}\left(\lambda_{i}\right)^{-\beta_{i}}+\mu_{i} m c_{i}\right)\left(n_{i}+\exp \left(n_{i} / 4\right)\right)\right]
$$

Asymptotic system availability

$$
A_{\mathrm{s}}(n, \lambda, \mu)=\prod_{i=1}^{m}\left[1-\left(1-\frac{\mu_{i}}{\lambda_{i}+\mu_{i}}\right)^{n_{i}}\right]
$$

System design configuration

$$
\begin{aligned}
& \sum_{i=1}^{m} p_{i}\left(n_{i}\right)^{2} \leq D_{1} \\
& \sum_{i=1}^{m} w_{i} n_{i} \exp \left(n_{i} / 4\right) \leq D_{2} \\
& n_{i} \geq 1\left(n_{i} \in \mathbb{Z}^{+}\right) \\
& \lambda_{i} \in\left[\lambda_{i}^{L}, \lambda_{i}^{U}\right] \subset \Re^{+}, \mu_{i} \in\left[\mu_{i}^{L}, \mu_{i}^{U}\right] \subset \Re^{+} \\
& A_{\mathrm{s}} \geq A_{\mathrm{s}}^{*} \\
& C_{s} \leq C_{s}^{*}
\end{aligned}
$$

where $C_{\mathrm{s}}(\bullet)$ is the total system cost, $n_{i}$ is the number of identical redundant components to be used in the $i$ th subsystem, $\lambda_{i}$ is the failure rate of the components in the $i$ th subsystem, $\mu_{i}$ is the repair rate of the components in the $i$ th subsystem, $m$ is the total number of subsystems in the system. $\beta_{i}$ and $\alpha_{i}$ are parameters representing the physical features of each component in each subsystem $i . p_{i}$ is the product of weight and volume per component in each subsystem $i$, and $w_{i}$ is the weight of one component in each subsystem $i . D_{1}, D_{2},\left(A_{\mathrm{s}}^{*}\right.$ and $C_{\mathrm{s}}^{*}$ are the design limits. $\lambda_{i}^{L}$, $\mu_{i}{ }^{L}, \lambda_{i}{ }^{U}$ and $\mu_{i}{ }^{U}$ are the lower and upper bounds for the failure and repair rates, respectively. It should be noted that the weight constraint given in Eq. (4) is increased by the interconnecting links modeled by $\exp \left(n_{i} / 4\right)$. 
In [18-20], the authors solved the problem by considering the system availability as a single objective problem, whereas in [21] the objectives of system availability and cost have been converted into a single objective by resorting to two weighted sum methods. In the present work, both objectives are considered in a Pareto front. Therefore, the above problem is formulated as follows:

Minimize $C_{\mathrm{s}}(n, \lambda, \mu)$

Maximize $A_{\mathrm{s}}(n, \lambda, \mu)$

subject to

Equations (3)-(5)

\section{Multi-objective Strawberry Algorithm}

First introduced by Salhi and Fraga [24], the strawberry algorithm also known as the Plant Propagation Algorithm and propagate. Typically, a good place is one which is sunny, has enough nutrients and humidity. Note that we are not explicitly concerned with these growth factors. To improve its chances of survival in nature, a strawberry plant implements a very basic strategy which is:

1. In a good spot, send many short runners (exploitation);

2. In a poor spot, send few long runners (exploration).

This strategy which is not unique to the strawberry plant can be implemented for any type of optimization/search problem including those involving two or more objective functions. For $\min f(x)$, where $f(x)$ is a vector function, PPA can be described as shown in Algorithm 1 [25].

Algorithm 1 Pseudo-code of the implemented MOPPA [25].

Only $N$, the population size has to be set arbitrarily and possibly the number of generations for the stopping crite-

Algorithm 1. Pseudo-code of the implemented MOPPA [25].

0 : Given: $f(x)$; a vector function; $n_{g}$ : number of generations to perform; $n_{p}$ : the propagation size; $n_{r}$ : maximum number of runners to propagate:

1: Output: $z$ : vector approximation to Nondominated frontier:

$2 \quad p \leftarrow$ initial random population of size $n_{p}$

3 for $n_{g}$ generations do

$4 \quad$ prune population $p$; removing similar solutions

$5 \quad N \leftarrow$ fitness $(p)$

$>$ Use rank based fitness

$p<\phi$

$>$ Empty set

7 :

8:

for $i \leftarrow 0 \ldots n_{p}$ do

$x \leftarrow \operatorname{select}(p ; N) \quad>$ Tournament fitness based selection

9:

10:

for each runner to generate do $>$ Nbre prop. to fitness rounded up

$x^{\prime} \leftarrow$ new solution $(x ; 1-N) \quad>$ Distance inversely proportional to

fitness

11: $\quad p \leftarrow x^{\prime} \cup \mathrm{p}$

$>$ Add to new population

12: $\quad$ endfor

14: endfor

15:

16:

$p \leftarrow \mathrm{p} \cup$ Nondominated $(p)$

$>$ New population with elitism

endfor

17: $\quad z \leftarrow \operatorname{Nondominated}(p)$

or PPA is a Nature-Inspired heuristic that emulates the way plants and in particular the strawberry plant propagation. The strawberry plant can propagate through seeds, but as a hybrid, it relies more on runners. A runner is a long branch that grows over ground. When it touches the ground, it produces roots which then give rise to another strawberry plant. Strawberry plants use runners to explore the landscape where they happen to be to find good places to grow rion. So, PPA only requires two arbitrary parameters. Compare this with the seven parameters that the genetic algorithm implementation requires and the five parameters that the simulated annealing implementation requires [26].

PPA has been shown to be competitive in continuous global optimization [27]. It has also been shown to work well on discrete optimization problems such as the TSP [26]. A variant of PPA which emulates propagation by 
seeds has been implemented and shown to work also very well on continuous problems [28]. Another variant that combines propagation via runners and seeds has also been introduced in $[29,30]$.

From its inception, PPA [24] has been considered for multi-objective optimization. Indeed, in [25], a bi-criterion optimization problem arising in chemical engineering has been considered. The two objectives were combined in an additive fashion using a couple of parameters $\lambda_{1}$ and $\lambda_{2}$ such that $\lambda_{1}+\lambda_{2}=1$. Here, we are concerned with the design of the parallel-series system problem (see Fig. 1). As described in Sect. 2, it involves two objectives namely the cost and the availability. The issue is to optimize with respect to these two conflicting objectives with the aim of providing the system designer with an optimum or near optimum decision. This decision is found in a Pareto Front which is a set of potential solutions. An approach to choosing the best solution from the PF is described below.

\section{Best Compromise Solution}

Solving multi-objective optimization problems is confronting its main disadvantage which is the Pareto Front (PF). PF is a set of optimal solutions and selecting a single solution is challenging for the decision maker. Several works have been devoted to the development of methods allowing the selection of the best compromise (called also preferred) solutions. An overview of these methods is listed in [31-36].

In this paper, the fuzzy set method is used to determine the best compromise solution from the obtained PF. When the multi-objective strawberry algorithm has generated the $\mathrm{PF}$, the following algorithm is implemented [35-38]:

- Perform fuzzy-based mechanisms:

Table 1 Data of the system

\begin{tabular}{llllll}
\hline Subsystem $i$ & $\alpha_{i}\left(10^{-5}\right)$ & $\beta_{i}$ & $m c_{i}$ & $p_{i}$ & $w_{i}$ \\
\hline 1 & 1.25 & 1.5 & 500 & 2 & 6 \\
2 & 2.70 & 1.5 & 500 & 4 & 9 \\
3 & 8.10 & 1.5 & 500 & 3 & 7 \\
4 & 4.50 & 1.5 & 500 & 2 & 6 \\
5 & 1.90 & 1.5 & 500 & 4 & 8 \\
6 & 3.55 & 1.5 & 500 & 2 & 5 \\
7 & 2.45 & 1.5 & 500 & 4 & 3 \\
8 & 6.30 & 1.5 & 500 & 3 & 9 \\
9 & 1.80 & 1.5 & 500 & 2 & 7 \\
10 & 5.25 & 1.5 & 500 & 2 & 5 \\
\hline
\end{tabular}

Table 2 Number of non-dominated solutions (Conditions 1)

\begin{tabular}{ll}
\hline No. & Number of non-dominated solutions \\
\hline 1 & 36 \\
2 & 49 \\
3 & 51 \\
4 & 51 \\
5 & 72 \\
6 & 56 \\
7 & 82 \\
8 & 62 \\
9 & 58 \\
10 & 53 \\
\hline
\end{tabular}

Table 3 Number of non-dominated solutions (Conditions 2)

\begin{tabular}{ll}
\hline No. & Number of non-dominated solutions \\
\hline 1 & 49 \\
$\mathbf{2}$ & $\mathbf{9 1}$ \\
3 & 84 \\
4 & 39 \\
5 & 47 \\
6 & 49 \\
7 & 83 \\
8 & 40 \\
9 & 37 \\
10 & 50 \\
\hline
\end{tabular}

Bold indicates the Highest number of non-dominated solutions

For system cost (minimizing function),

$$
\mu_{j}=\left\{\begin{array}{l}
1, F_{j} \leq F_{j}^{\min } \\
\frac{F_{j}^{\max }-F_{j}}{F_{j}^{\max }-F_{j}^{\min }}, F_{j}^{\min }<F_{j}<F_{j}^{\max } \\
0, F_{j} \geq F_{j}^{\max }
\end{array}\right.
$$

For system availability (maximizing function),

$$
\mu_{j}=\left\{\begin{array}{l}
0, F_{j} \leq F_{j}^{\min } \\
\frac{F_{j}-F_{j}^{\min }}{F_{j}^{\max }-F_{j}^{\min }}, F_{j}^{\min }<F_{j}<F_{j}^{\max } \\
1, F_{j} \geq F_{j}^{\max }
\end{array}\right.
$$

where $\mu_{j}$ is the membership function, $F_{j}$ is the $j$ th objective function, and $\left(F_{j}^{\min }, F_{j}^{\max }\right)$ are its minimum and maximum values, respectively.

- Calculate the normalized membership value: 
Table 4 Best Pareto front

\begin{tabular}{|c|c|c|c|c|c|}
\hline No. & $C_{\mathrm{s}}$ & $A_{\mathrm{s}}$ & No. & $C_{\mathrm{s}}$ & $A_{\mathrm{s}}$ \\
\hline 1 & 248.0301 & 0.9675 & 51 & 226.6152 & 0.9508 \\
\hline 2 & 228.6186 & 0.9541 & 52 & 227.0615 & 0.9534 \\
\hline 3 & 199.0064 & 0.9167 & 53 & 195.7784 & 0.9098 \\
\hline 4 & 208.2397 & 0.9307 & 54 & 219.7293 & 0.9459 \\
\hline 5 & 233.2392 & 0.9601 & 55 & 210.9245 & 0.9344 \\
\hline 6 & 210.8452 & 0.9322 & 56 & 247.0194 & 0.9672 \\
\hline 7 & 230.3806 & 0.9568 & 57 & 195.1605 & 0.9077 \\
\hline 8 & 202.3160 & 0.9195 & 58 & 219.5095 & 0.9452 \\
\hline 9 & 246.4674 & 0.9671 & 59 & 245.1320 & 0.9653 \\
\hline 10 & 232.6731 & 0.9571 & 60 & 241.7951 & 0.9645 \\
\hline 11 & 209.8697 & 0.9317 & 61 & 230.3806 & 0.9568 \\
\hline 12 & 241.4323 & 0.9640 & 62 & 241.2529 & 0.9638 \\
\hline 13 & 245.1320 & 0.9653 & 63 & 234.0463 & 0.9603 \\
\hline 14 & 247.3743 & 0.9673 & 64 & 216.5461 & 0.9412 \\
\hline 15 & 235.7759 & 0.9617 & 65 & 198.9011 & 0.9148 \\
\hline 16 & 209.8697 & 0.9317 & 66 & 195.1605 & 0.9077 \\
\hline 17 & 245.5489 & 0.9669 & 67 & 202.7490 & 0.9215 \\
\hline 18 & 203.8581 & 0.9263 & 68 & 210.8452 & 0.9322 \\
\hline 19 & 240.9682 & 0.9637 & 69 & 238.5180 & 0.9628 \\
\hline 20 & 245.5489 & 0.9669 & 70 & 240.7870 & 0.9634 \\
\hline 21 & 233.0069 & 0.9581 & 71 & 240.9682 & 0.9637 \\
\hline 22 & 223.7531 & 0.9483 & 72 & 245.3488 & 0.9658 \\
\hline 23 & 218.2685 & 0.9413 & 73 & 227.0615 & 0.9534 \\
\hline 24 & 246.4674 & 0.9671 & 74 & 206.9551 & 0.9291 \\
\hline 25 & 240.8994 & 0.9636 & 75 & 239.6384 & 0.9631 \\
\hline 26 & 226.6152 & 0.9508 & 76 & 232.6731 & 0.9571 \\
\hline 27 & 195.7784 & 0.9098 & 77 & 216.5461 & 0.9412 \\
\hline 28 & 241.7951 & 0.9645 & 78 & 202.7490 & 0.9215 \\
\hline 29 & 193.2516 & 0.9043 & 79 & 240.8994 & 0.9636 \\
\hline 30 & 235.7759 & 0.9617 & 80 & 212.9021 & 0.9349 \\
\hline 31 & 233.0069 & 0.9581 & 81 & 233.0069 & 0.9581 \\
\hline 32 & 223.7531 & 0.9483 & 82 & 199.0064 & 0.9167 \\
\hline 33 & 218.2685 & 0.9413 & 83 & 208.2397 & 0.9307 \\
\hline 34 & 246.4674 & 0.9671 & 84 & 224.3092 & 0.9495 \\
\hline 35 & 240.8994 & 0.9636 & 85 & 234.0463 & 0.9603 \\
\hline 36 & 226.6152 & 0.9508 & 86 & 247.0194 & 0.9672 \\
\hline 37 & 195.7784 & 0.9098 & 87 & 243.9883 & 0.9651 \\
\hline 38 & 241.7951 & 0.9645 & 88 & 245.3488 & 0.9658 \\
\hline 39 & 193.2516 & 0.9043 & 89 & 193.2516 & 0.9043 \\
\hline 40 & 235.7759 & 0.9617 & 90 & 243.9883 & 0.9651 \\
\hline 41 & 198.9011 & 0.9148 & 91 & 197.1770 & 0.9147 \\
\hline 42 & 219.7293 & 0.9459 & & & \\
\hline 43 & 202.3160 & 0.9195 & & & \\
\hline 44 & 241.2529 & 0.9638 & & & \\
\hline 45 & 203.8581 & 0.9263 & & & \\
\hline 46 & 212.9021 & 0.9349 & & & \\
\hline 47 & 228.6186 & 0.9541 & & & \\
\hline 48 & 206.9551 & 0.9291 & & & \\
\hline
\end{tabular}

Table 4 continued

\begin{tabular}{llllll}
\hline No. & $C_{\mathrm{s}}$ & $A_{\mathrm{s}}$ & No. & $C_{\mathrm{s}}$ & $A_{\mathrm{s}}$ \\
\hline 49 & 241.4323 & 0.9640 & & & \\
50 & 218.2685 & 0.9413 & & & \\
\hline & \\
$\mu^{k}=\sum_{j=1}^{2} \mu_{j}^{k}$ & & & \\
$\sum_{l=1}^{M} \sum_{j=1}^{2} \mu_{j}^{l}$ & & &
\end{tabular}

where $M$ is the number of solutions in PF. The solution having the maximum value of $\mu^{k}$ represents the best compromise solution.

\section{Case Study}

The parallel-series system (see Fig. 1) studied here contains 10 subsystems. Table 1 reports its data. The limits of the system design are: $C_{\mathrm{s}}^{*}=250 ; D_{1}=200 ; D_{2}=300$, in arbitrary units, and $A_{\mathrm{s}}=0.9$ [20,21]. The lower and upper bounds for the design variables are: $n_{i} \geq 1\left(n_{i} \in \mathbb{Z}^{+}\right), \lambda_{i} \in$ $\left[10^{-7}, 10^{-3}\right] \subset \mathbb{R}^{+}$, and $\mu_{i} \in\left[32 \times 10^{-7}, 32 \times 10^{-3}\right] \subset \mathbb{R}^{+}$.

\section{Results and Discussion}

The multi-objective strawberry algorithm and fuzzy set method have been encoded using MATLAB 2017 and run on a PC (Intel Core I5-7300U vPro 7th Gen, $2.7 \mathrm{GHz}$, $8 \mathrm{~GB}$ of RAM). The optimization algorithm has been run under two values of the number of runners ( 3 and 4 ) with the same population size (100) and maximum number of iterations (200). These values are called Conditions 1 and Conditions 2, respectively. The above parameters have been fixed by trial-and-error and based on experience. Ten independent runs have been performed with each value of number of runners in order to select the run with the highest number of non-dominated solutions. From Tables 2 and 3, it can be observed that the maximum number of nondominated solutions is 91 and has been obtained with four runners. The consumed CPU time was 6.9165 s. Figure 2 shows this Pareto front, whereas the values of the system cost and availability of the 91 solutions are reported in Table 4 . The points are relatively extensive on front.

The normalized membership value has been calculated for each value of Table 4 and is reported in Table 5. From this table, it can be observed that the maximum value is 


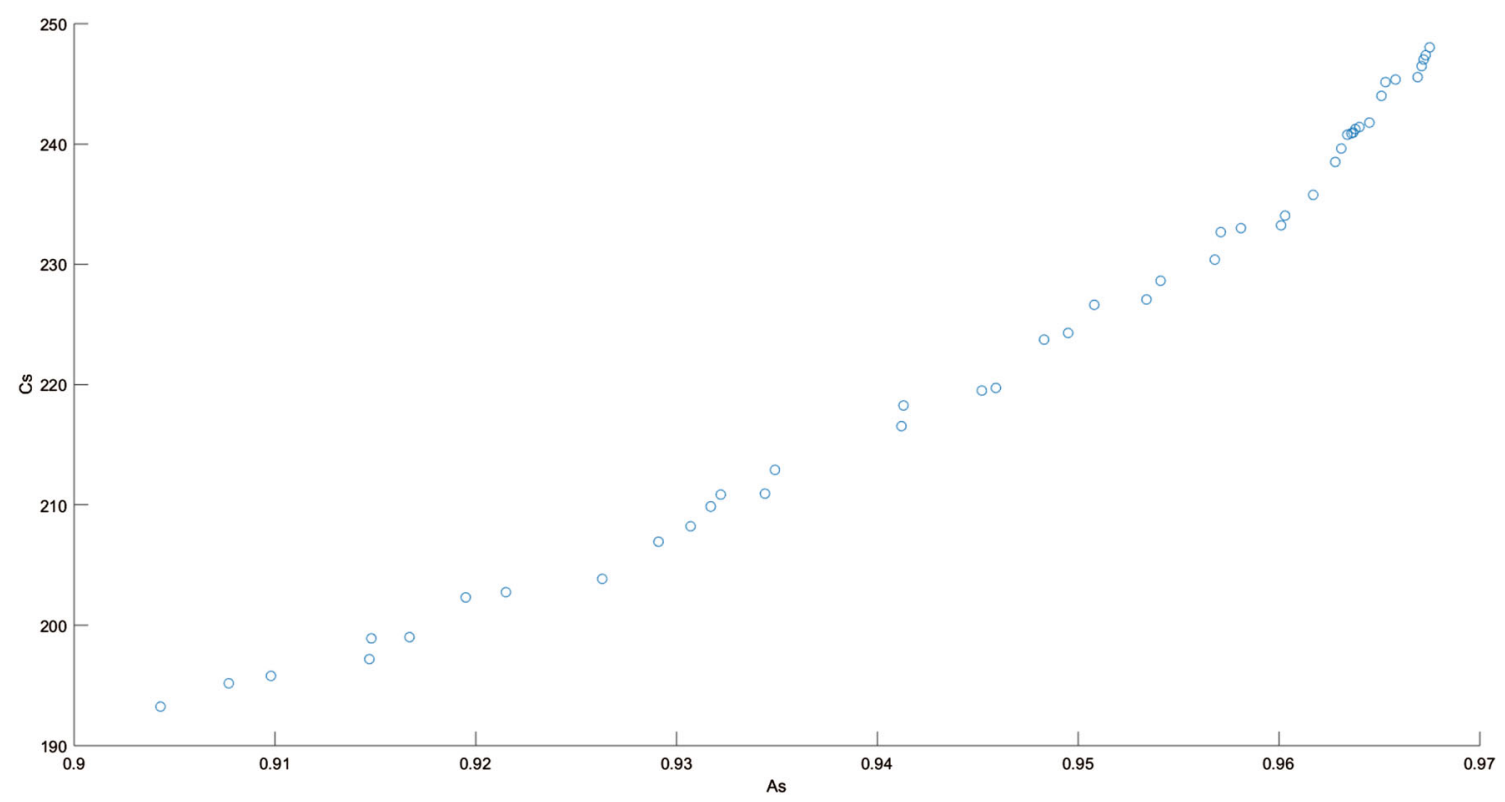

Fig. 2 Best Pareto front

those of the solutions 42 and $52\left(\mu^{k}=0.0117856\right)$. The related solutions represent the best compromise solutions of the obtained PF. The values of the decision variables of these solutions are given in Table 6. Therefore, this is the best compromise solution for the decision maker, where the system cost and availability are 219.7293 and 0.9459 , respectively.

\section{Conclusions}

In this work, the multi-objective system availability and cost have been investigated. The problem has been solved by using the multi-objective strawberry algorithm which requires a few parameters. It was the first implementation of this efficient algorithm to solve this kind of problem. A fuzzy method has been used to determine the best compromise solution from the Pareto front for helping the decision maker. A numerical case study consisting of a system with 10 subsystems has been solved in order to highlight the applicability of the proposed solution approach.

In the future, this proposed approach will be hybridized with other methods to provide better performance metrics. 
Table 5 Values of the normalized membership

\begin{tabular}{|c|c|c|c|}
\hline No. & $\mu^{k}$ & No. & $\mu^{k}$ \\
\hline 1 & 0.0100301 & 51 & 0.0112983 \\
\hline 2 & 0.0114616 & 52 & 0.0116248 \\
\hline 3 & 0.0109487 & 53 & 0.0104333 \\
\hline 4 & 0.0114702 & 54 & 0.0117856 \\
\hline 5 & 0.0115622 & 55 & 0.0115671 \\
\hline 6 & 0.0112388 & 56 & 0.0101616 \\
\hline 7 & 0.0115565 & 57 & 0.0102174 \\
\hline 8 & 0.0107814 & 58 & 0.0117108 \\
\hline 9 & 0.0102555 & 59 & 0.0102107 \\
\hline 10 & 0.0111854 & 60 & 0.0106976 \\
\hline 11 & 0.0113291 & 61 & 0.0115565 \\
\hline 12 & 0.0106830 & 62 & 0.0106878 \\
\hline 13 & 0.0102107 & 63 & 0.0114469 \\
\hline 14 & 0.0101119 & 64 & 0.0116146 \\
\hline 15 & 0.0113554 & 65 & 0.0106559 \\
\hline 16 & 0.0113291 & 66 & 0.0102174 \\
\hline 17 & 0.0103917 & 67 & 0.0110212 \\
\hline 18 & 0.0115771 & 68 & 0.0112388 \\
\hline 19 & 0.0107117 & 69 & 0.0110275 \\
\hline 20 & 0.0103917 & 70 & 0.0107073 \\
\hline 21 & 0.0112829 & 71 & 0.0107117 \\
\hline 22 & 0.0114236 & 72 & 0.0102476 \\
\hline 23 & 0.0113192 & 73 & 0.0116248 \\
\hline 24 & 0.0102555 & 74 & 0.0114560 \\
\hline 25 & 0.0107202 & 75 & 0.0108589 \\
\hline 26 & 0.0112983 & 76 & 0.0111854 \\
\hline 27 & 0.0104333 & 77 & 0.0116146 \\
\hline 28 & 0.0106976 & 78 & 0.0110212 \\
\hline 29 & 0.0100301 & 79 & 0.0107202 \\
\hline 30 & 0.0113554 & 80 & 0.0112912 \\
\hline 31 & 0.0110275 & 81 & 0.0112829 \\
\hline 32 & 0.0117108 & 82 & 0.0109487 \\
\hline 33 & 0.0109577 & 83 & 0.0114702 \\
\hline 34 & 0.0100301 & 84 & 0.0115134 \\
\hline 35 & 0.0115134 & 85 & 0.0114469 \\
\hline 36 & 0.0107073 & 86 & 0.0101616 \\
\hline 37 & 0.0114236 & 87 & 0.0103821 \\
\hline 38 & 0.0115622 & 88 & 0.0102476 \\
\hline 39 & 0.0115671 & 89 & 0.0100301 \\
\hline 40 & 0.0109577 & 90 & 0.0103821 \\
\hline 41 & 0.0106559 & 91 & 0.0109632 \\
\hline 42 & 0.0117856 & & \\
\hline 43 & 0.0107814 & & \\
\hline 44 & 0.0106878 & & \\
\hline 45 & 0.0115771 & & \\
\hline 46 & 0.0112912 & & \\
\hline 47 & 0.0114616 & & \\
\hline 48 & 0.0114560 & & \\
\hline
\end{tabular}

Table 5 continued

\begin{tabular}{lll}
\hline No. & $\mu^{k}$ & No. \\
\hline 49 & 0.0106830 & $\mu^{k}$ \\
50 & 0.0113192 & \\
\hline Bold indicates the Highest normalized membership value
\end{tabular}

Table 6 Best compromise solution

\begin{tabular}{rrrrr}
\hline$n$ & \multicolumn{1}{c}{$\lambda\left(10^{-3}\right)$} & $\mu\left(10^{-2}\right)$ & \multicolumn{1}{c}{$C_{s}$} & \multicolumn{1}{c}{$A_{s}$} \\
\hline 3,2, & $(0.5178,0.5046$, & $(0.27249$, & 219.7293 & 0.9459 \\
2,3, & $0.6814,0.9439$, & 0.76399, & & \\
2,3, & $0.6741,0.9603$, & 0.84547, & & \\
3,2, & $0.7212,0.7156$, & 0.61919, & & \\
$2,2)$ & 0.8516, & 0.69907, & & \\
& $0.9156)$ & 0.43691, & & \\
& & 0.28401, & & \\
& & 0.71324, & & \\
& & 0.98624, & & \\
& & $1.56609)$ & & \\
\hline
\end{tabular}

Bold indicates the Highest normalized membership value

Acknowledgements We are grateful to ESRC, Grant ES/L011859/1, for partially funding this research.

Open Access This article is licensed under a Creative Commons Attribution 4.0 International License, which permits use, sharing, adaptation, distribution and reproduction in any medium or format, as long as you give appropriate credit to the original author(s) and the source, provide a link to the Creative Commons licence, and indicate if changes were made. The images or other third party material in this article are included in the article's Creative Commons licence, unless indicated otherwise in a credit line to the material. If material is not included in the article's Creative Commons licence and your intended use is not permitted by statutory regulation or exceeds the permitted use, you will need to obtain permission directly from the copyright holder. To view a copy of this licence, visit http://creativecommons. org/licenses/by/4.0/.

\section{References}

1. Marseguerra, M., Zio, E., Martorell, S.: Basics of genetic algorithms optimization for RAMS applications. Reliab. Eng. Syst. Saf. 91, 977-991 (2006). https://doi.org/10.1016/j.ress.2005.11. 046

2. Torres-Echeverría, A.C., Martorell, S., Thompson, H.A.: Design optimization of a safety-instrumented system based on RAMS +C addressing IEC 61508 requirements and diverse redundancy. Reliab. Eng. Syst. Saf. 94, 162-179 (2009). https://doi.org/10. 1016/j.ress.2008.02.010

3. Mellal, M.A., Zio, E.: An adaptive particle swarm optimization method for multi-objective system reliability optimization. J. Risk Reliab. 233, 990-1001 (2019). https://doi.org/10.1177/ 1748006X19852814 
4. Mellal, M.A., Zio, E.: An adaptive cuckoo optimization algorithm for system design optimization under failure dependencies. J. Risk Reliab. 233, 1099-1105 (2019)

5. Chebouba, B.N., Mellal, M.A., Adjerid, S.: Multi-objective system reliability optimization in a power plant. In: The 3rd International Conference on Electrical Sciences and Technologies in Maghreb. Algiers, Algeria (2018)

6. Coit, D.W., Smith, A.E.: Reliability optimization of series-parallel systems using a genetic algorithm. IEEE Trans. Reliab. 45, 254-260, 266 (1996). https://doi.org/10.1109/24.510811

7. Chen, T.C.: IAs based approach for reliability redundancy allocation problems. Appl. Math. Comput. 182, 1556-1567 (2006). https://doi.org/10.1016/j.amc.2006.05.044

8. Hsieh, Y.C., You, P.S.: An effective immune based two-phase approach for the optimal reliability-redundancy allocation problem. Appl. Math. Comput. 218, 1297-1307 (2011)

9. Garg, H., Rani, M., Sharma, S.P.: An efficient two phase approach for solving reliability-redundancy allocation problem using artificial bee colony technique. Comput. Oper. Res. 40, 2961-2969 (2013). https://doi.org/10.1016/j.cor.2013.07.014

10. Garg, H.: An approach for solving constrained reliability-redundancy allocation problems using cuckoo search algorithm. BeniSuef Univ. J. Basic Appl. Sci. 4, 14-25 (2015). https://doi.org/10. 1016/j.bjbas.2015.02.003

11. Mellal, M.A., Zio, E.: A penalty guided stochastic fractal search approach for system reliability optimization. Reliab. Eng. Syst. Saf. (2016). https://doi.org/10.1016/j.ress.2016.03.019

12. Mellal, M.A., Zio, E.: System reliability-redundancy allocation by evolutionary computation. In: 2017 2nd International Conference on System Reliability and Safety. pp. 15-19. IEEE, Milan, Italy (2017)

13. Mellal, M.A., Williams, E.J.: Large scale reliability-redundancy allocation optimization problem using three soft computing methods. In: Modeling and Simulation based Analysis in Reliability Engineering, pp. 199-214. CRC Press, Francis \& Taylor, Boca Raton (2018)

14. Huang, X., Coolen, F.P.A., Coolen-Maturi, T.: A heuristic survival signature based approach for reliability-redundancy allocation. Reliab. Eng. Syst. Saf. (2019). https://doi.org/10.1016/j. ress.2019.02.010

15. Juybari, M.N., Abouei-Ardakan, M., Davari-Ardakani, H.: A penalty-guided fractal search algorithm for reliability-redundancy allocation problems with cold-standby strategy. Proc. Inst. Mech. Eng. Part O J. Risk Reliab. (2019). https://doi.org/10.1177/ 1748006X19825707

16. Mellal, M.A., Zio, E.: System reliability-redundancy optimization with cold-standby strategy by an enhanced nest cuckoo optimization algorithm. Reliab. Eng. Syst. Saf. 201, 106973 (2020). https://doi.org/10.1016/j.ress.2020.106973

17. Chebouba, B.N., Mellal, M.A., Adjerid, S.: Fuzzy multiobjective system reliability optimization by genetic algorithms and clustering analysis. Qual. Reliab. Eng. Int. (2020). https://doi.org/10. 1002/qre. 2809

18. Liu, G.S.: Availability optimization for repairable parallel-series system by applying Tabu-GA combination method. In: 10th IEEE International Conference on Industrial Informatics. pp. 803-808. Beijing, China (2012)

19. Liu, G.S.: Availability optimization for repairable n-stage standby system by applying Tabu-GA combination method. Int. J. Model. Optim. 3, 245-250 (2013)

20. Mellal, M.A., Zio, E.: Availability optimization of parallel-series system by evolutionary computation. In: 3rd International Conference on System Reliability and Safety, pp. 198-202. Barcelona, Spain (2018)

21. Mellal, M.A., Salhi, A.: Parallel-series system optimization by weighting sum methods and nature-inspired computing. In:
Applied Nature-Inspired Computing: Algorithms and Case Studies. Springer, Singapore (2020)

22. Mellal, M.A., Chebouba, B.N.: Cost and availability optimization of overspeed protection system in a power plant. In: 2019 Int. Conf. Adv. Electr. Eng., pp. 1-4 (2019). https://doi.org/10.1109/ ICAEE47123.2019.9015115

23. Hu, L., Yue, D., Li, J.: Availability analysis and design optimization for a repairable series-parallel system with failure dependencies. Int. J. Innov. Comput. Inf. Control. 8, 6693-6705 (2012). https://doi.org/10.4156/aiss.vol3

24. Salhi, A., Fraga, E.S.: Nature-inspired optimisation approaches and the new plant propagation algorithm. In: The International Conference on Numerical Analysis and Optimization (2011)

25. Rodman, A.D., Fraga, E.S., Gerogiorgis, D.: On the application of a nature-inspired stochastic evolutionary algorithm to constrained multi-objective beer fermentation optimisation. Comput. Chem. Eng. 108, 448-459 (2018). https://doi.org/10.1016/j. compchemeng.2017.10.019

26. Selamoğlu, B.İ, Salhi, A.: The plant propagation algorithm for discrete optimisation: The case of the travelling salesman problem. In: Yang, X.S. (ed.) Nature-Inspired Computation in Engineering, pp. 43-61. Springer, Cham (2016)

27. Sulaiman, M., Salhi, A., Selamoglu, B.I., Kirikchi, O.B.: A plant propagation algorithm for constrained engineering optimisation problems. Math. Probl. Eng. (2014). https://doi.org/10.1155/ 2014/627416

28. Sulaiman, M., Salhi, A.: A seed-based plant propagation algorithm: The feeding station model. Sci. World J. (2015). https:// doi.org/10.1155/2015/904364

29. Sulaiman, M., Salhi, A.: A hybridisation of runner-based and seed-based plant propagation algorithms. In: Yang, X.S. (ed.) Nature-Inspired Computation in Engineering, pp. 195-215. Springer, Cham (2016)

30. Sulaiman, M., Salhi, A., Kha, A., Shakoor, M., Khan, W.: On the theoretical analysis of the plant propagation algorithms. Math. Probl. Eng. (2018). https://doi.org/10.1155/2018/6357935

31. de la Fuente, D., Vega-Rodríguez, M.A., Pérez, C.J.: Automatic selection of a single solution from the Pareto front to identify key players in social networks. Knowl. Based Syst. (2018). https:// doi.org/10.1016/j.knosys.2018.07.018

32. Zio, E., Bazzo, R.: A comparison of methods for selecting preferred solutions in multiobjective decision making. Comput. Intell. Syst. Ind. Eng. (2012). https://doi.org/10.2991/978-9491216-77-0_2

33. Kong, X., Yong, C., Wang, C., Li, P., Yu, L., Chen, Y.: Multiobjective power supply capacity evaluation method for active distribution network in power market environment. Int. J. Electr. Power Energy Syst. 115, 105467 (2020)

34. Zio, E., Bazzo, R.: A clustering procedure for reducing the number of representative solutions in the Pareto Front of multiobjective optimization problems. Eur. J. Oper. Res. (2011). https://doi.org/10.1016/j.ejor.2010.10.021

35. Qu, B.Y., Zhu, Y.S., Jiao, Y.S., Wu, M.Y., Suganthan, P.N., Liang, J.J.: A survey on multi-objective evolutionary algorithms for the solution of the environmental/economic dispatch problems. Swarm Evol. Comput. 38, 1-11 (2018)

36. Abido, M.A.: Multiobjective evolutionary algorithms for electric power dispatch problem. IEEE Trans. Evol. Comput. 10, 315-329 (2006)

37. Sakawa, M., Yano, H., Yumine, T.: An interactive fuzzy satisficing method for multiobjective linear programming problems and its application. IEEE Trans. Syst. Man. Cybern. 17, 654-661 (1987)

38. Mellal, M.A., Pecht, M.: A multi-objective design optimization framework for wind turbines under altitude consideration. Energy 
Convers. Manag. 222, 113212 (2020). https://doi.org/10.1016/j. enconman.2020.113212

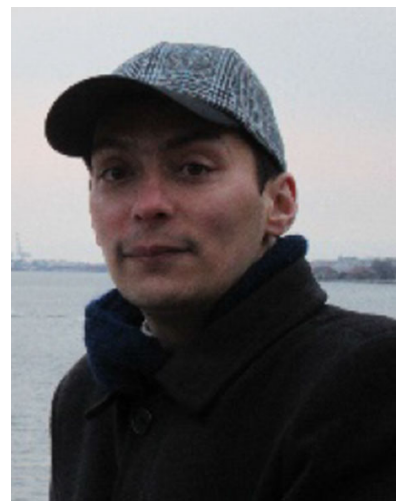

Mohamed Arezki Mellal is an Associate Professor at the Department of Mechanical Engineering, Faculty of Technology, M'Hamed Bougara University, Algeria and was a Visiting Scholar at the Center for Advanced Life Cycle Engineering, Department of Mechanical Engineering, University of Maryland, College Park, MD, USA. Likewise, he was a Visiting Scholar at various universities. He has published in several journals and conference proceedings. He has edited five books and authored seven book chapters. He is a member of the Algerian National Laboratory for Maintenance Education in conjunction with the European Union (Erasmus+). He has also been a committee member for over seventy international conferences. He serves as a regular reviewer for eighteen SCI-indexed journals and an editorial board member in seven peerreviewed international journals.

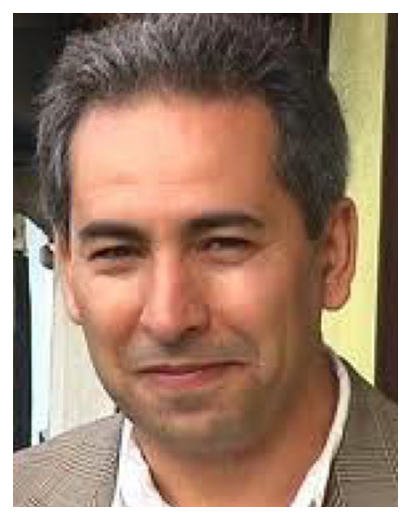

Abdellah Salhi is a Professor of Operational Research at the University of Essex, UK. He studied for a Ph.D. at Aston University, in Birmingham, UK and thereafter, worked as a Research Fellow at the Universities of Leeds, Edinburgh, and Southampton, UK, before settling in Essex. His research work covers a variety of topics in OR and Data Science. He designed the Plant Propagation Algorithm and applied it to a number of challenging problems in design and optimization. His most recent work includes the design and implementation of a new approach to protecting the security of data. He has lead many research projects concerned with operations at container ports, green distribution in online shopping, smart town centres, optimum pollination with honeybees and others. His work appears in a number of prominent journals such as the ACM Transactions in Evolutionary Computation, the Annals of Operations Research, the Journal of the Operational Research Society, Computers and Operations Research, to name a few. He co-organizes the yearly EGL Workshop at one of the universities of Essex, Greenwich and UCL. He has been the Head of the Department of Mathematical Sciences of Essex University from 2010 to 2016. 